\title{
Intuitionistic fuzzy strong hyper K-subalgebras in Hyper K-algebras
}

\author{
Chul Hwan Park \\ Department of Mathematics, University of Ulsan, Ulsan 680-749, Korea
}

\begin{abstract}
Intuitionistic fuzzifications of (strong) hyper K-subalgebras in hyper K-algebras are discussed, and related properties are investigated. Relations between intuitionistic fuzzy hyper $\mathrm{K}$-subalgebras and intuitionistic fuzzy strong hyper Ksubalgebras are provided.
\end{abstract}

Key words : Intuitionistic fuzzy (strong) hyper K-subalgebra.

\section{Introduction}

The study of BCK-algebras was initiated by K. Iséki in 1966 as a generalization of the concept of set-theoretic difference and propositional calculus. Since then many researches worked in this area. The hyperstructure theory (called also multialgebras) is introduced in 1934 by $\mathrm{F}$. Marty [12] at the 8th congress of Scandinavian Mathematiciens. Around the 40's, several authors worked on hypergroups, especially in France and in the United States, but also in Italy, Russia, Japan and Iran. Hyperstructures have many applications to several sectors of both pure and applied sciences. Recently in [11] Y. B. Jun et al. introduced and studied hyper $B C K$-algebra which is a generalization of a BCK-algebra. In [1] and [11] R. A. Borzooei et al. constructed the hyper K-algebras, and studied (weak) implicative hyper K-ideals in hyper K-algebras. In [9] and [10] Y. B. Jun et al. studied the fuzzy (implicative) hyper $\mathrm{K}$-ideals in hyper K-algebras. Y. B. Jun et al. [8] introduced the notion of fuzzy (weak) implicative hyper K-ideals, and investigated related properties. They gave relations among fuzzy weak implicative hyper K-ideals, fuzzy implicative hyper K-ideals, and fuzzy hyper K-ideals. In [4], R. A. Borzooei and Y. B. Jun studied intuitionistic fuzzy hyper BCKideals of hyper BCK-algebras. In [2], R. A. Borzooei and Y. B. Jun discussed intuitionistic fuzzifications of (weak) implicative hyper $\mathrm{K}$-ideals in hyper K-algebras. They gave relations among intuitionistic fuzzy hyper $\mathrm{K}$-ideals, intuitionistic fuzzy weak hyper K-ideals, intuitionistic fuzzy implicative hyper $\mathrm{K}$-ideals and intuitionistic fuzzy weak implicative hyper $\mathrm{K}$-ideals. They provided conditions for an intuitionistic fuzzy hyper K-ideal to be an intuitionistic fuzzy implicative hyper K-ideal, and also discussed condi- tions for an intuitionistic fuzzy weak hyper K-ideal to be an intuitionistic fuzzy weak implicative hyper K-ideal. In this paper we consider the intuitionistic fuzzifications of (strong) hyper K-subalgebras in hyper $\mathrm{K}$-algebras. We give relations between intuitionistic fuzzy hyper K-subalgebras and intuitionistic fuzzy strong hyper K-subalgebras.

\section{Preliminaries}

We include some elementary aspects of hyper Kalgebras that are necessary for this paper, and for more details we refer to [3] and [13]. Let $H$ be a non-empty set endowed with a hyper operation " $O$ ", that is, $\circ$ is a function from $H \times H$ to $\mathcal{P}^{*}(H)=\mathcal{P}(H) \backslash\{\emptyset\}$. For two subsets $A$ and $B$ of $H$, denote by $A \circ B$ the set $\bigcup_{a \in A, b \in B} a \circ b$.

By a hyper I-algebra we mean a non-empty set $H$ endowed with a hyper operation " $O$ " and a constant 0 satisfying the following axioms:

(H1) $(x \circ z) \circ(y \circ z) \prec x \circ y$,

(H2) $(x \circ y) \circ z=(x \circ z) \circ y$,

(H3) $x \prec x$,

(H4) $x \prec y$ and $y \prec x$ imply $x=y$

for all $x, y, z \in H$, where $x \prec y$ is defined by $0 \in x \circ y$ and for every $A, B \subseteq H, A \prec B$ is defined by $\exists a \in A$ and $\exists b \in B$ such that $a \prec b$. If a hyper I-algebra $(H, 0,0)$ satisfies an additional condition:

(H5) $0 \prec x$ for all $x \in H$, 
then $(H, \circ, 0)$ is called a hyper K-algebra (see [3]).

In a hyper I-algebra $H$, the following hold (see [3, Proposition 3.4]):

(a1) $(A \circ B) \circ C=(A \circ C) \circ B$

(a2) $x \circ(x \circ y) \prec y$.

(a3) $x \circ y \prec z \Leftrightarrow x \circ z \prec y$.

(a4) $A \circ B \prec C \Leftrightarrow A \circ C \prec B$.

(a5) $(x \circ z) \circ(x \circ y) \prec y \circ z$.

(a6) $(A \circ C) \circ(B \circ C) \prec A \circ B$.

(a7) $A \circ(A \circ B) \prec B$.

(a8) $A \prec A$.

(a9) $A \subseteq B$ implies $A \prec B$.

for all $x, y, z \in H$ and for all nonempty subsets $A, B$ and $C$ of $H$.

Let $S$ be a subset of a hyper K-algebra $(H, 0,0)$ containing 0 . If $S$ is a hyper K-algebra with respect to the hyper operation " $O$ " on $H$, we say that $S$ is a hyper $K$-subalgebra of $(H, \circ, 0)$ (see [3]).

Lemma 2.1. [3] Let $S$ be a nonempty subset of $H$. Then $S$ is a hyper $\mathrm{K}$-subalgebra of $H$ if and only if $x \circ y \subseteq S$ for all $x, y \in S$.

\section{Intuitionistic fuzzy strong hyper K-subalgebras}

In what follows let $H$ denote a hyper $\mathrm{K}$-algebra unless otherwise specified. An intuitionistic fuzzy set (IFS, for short) in $H$ is an expression $\alpha$ given by

$$
\alpha=\left\{\left\langle x, \mu_{\alpha}(x), \gamma_{\alpha}(x)\right\rangle \mid x \in H\right\}
$$

where the functions $\mu_{\alpha}: H \rightarrow[0,1]$ and $\gamma_{\alpha}: H \rightarrow[0,1]$ denote the degree of membership (namely $\mu_{\alpha}(x)$ ) and the degree of nonmembership (namely $\gamma_{\alpha}(x)$ ) of each element $x \in H$ to $\alpha$, respectively, and

$$
0 \leq \mu_{\alpha}(x)+\gamma_{\alpha}(x) \leq 1
$$

for all $x \in H$. For the sake of simplicity, we shall use the notation $\alpha=\left\langle H, \mu_{\alpha}, \gamma_{\alpha}\right\rangle$ instead of $\alpha=$ $\left\{\left\langle x, \mu_{\alpha}(x), \gamma_{\alpha}(x)\right\rangle \mid x \in H\right\}$. Let $\alpha=\left\langle H, \mu_{\alpha}, \gamma_{\alpha}\right\rangle$ be an IFS in $H$ and let $m, n \in[0,1]$ with $m+n \leq 1$. Then the IFS $C_{(m, n)}$ in $H$ is defined by $C_{(m, n)}(x)=(m, n)$, i.e., $\mu_{C_{(m, n)}}(x)=m$ and $\gamma_{C_{(m, n)}}(x)=n$ for all $x \in H$. The representation " $\alpha(x) \geq(m, n)$ " means that $\mu_{\alpha}(x) \geq m$ and $\gamma_{\alpha}(x) \leq n$. Then the set $H_{\alpha}^{(m, n)}:=\{x \in H \mid \alpha(x) \geq$ $\left.C_{(m, n)}(x)\right\}=\left\{x \in H \mid \mu_{\alpha}(x) \geq m, \gamma_{\alpha}(x) \leq n\right\}$ is called an intuitionistic level set of $\alpha$ in $H$.
Definition 3.1. [6] An IFS $\alpha=\left\langle H, \mu_{\alpha}, \gamma_{\alpha}\right\rangle$ in $H$ is called an intuitionistic fuzzy hyper $K$-subalgebra of $H$ if it satisfies:

$$
\begin{aligned}
& \inf _{a \in x \circ y} \mu_{\alpha}(a) \geq \min \left\{\mu_{\alpha}(x), \mu_{\alpha}(y)\right\}, \\
& \sup _{b \in x \circ y} \gamma_{\alpha}(b) \leq \max \left\{\gamma_{\alpha}(x), \gamma_{\alpha}(y)\right\}
\end{aligned}
$$

for all $x, y \in H$.

Lemma 3.2. [6] An IFS $\alpha=\left\langle H, \mu_{\alpha}, \gamma_{\alpha}\right\rangle$ in $H$ is an intuitionistic fuzzy hyper $\mathrm{K}$-subalgebra of $H$ if and only if the nonempty intuitionistic level set $H_{\alpha}^{(m, n)}$ is a hyper $\mathrm{K}$ subalgebra of $H$ for all $m, n \in[0,1]$ with $m+n \leq 1$.

Definition 3.3. An IFS $\alpha=\left\langle H, \mu_{\alpha}, \gamma_{\alpha}\right\rangle$ in $H$ is called an intuitionistic fuzzy strong hyper $K$-subalgebra of $H$ if it satisfies:

$$
(\forall x, y \in H)\left(\inf _{a \in x \circ y} \mu_{\alpha}(a) \geq \mu_{\alpha}(x), \sup _{b \in x \circ y} \gamma_{\alpha}(b) \leq \gamma_{\alpha}(x)\right) .
$$

Example 3.4. Let $H=\{0, a, b\}$ be a hyper K-algebra with the following Cayley table:

\begin{tabular}{c|ccc}
$\circ$ & 0 & $a$ & $b$ \\
\hline 0 & $\{0\}$ & $\{0\}$ & $\{0\}$ \\
$a$ & $\{a\}$ & $\{0, a\}$ & $\{0, a\}$ \\
$b$ & $\{b\}$ & $\{a, b\}$ & $\{0, a, b\}$
\end{tabular}

Define an IFS $\alpha=\left\langle H, \mu_{\alpha}, \gamma_{\alpha}\right\rangle$ in $H$ by

$$
\alpha=\left\langle H,\left(\frac{0}{0.6}, \frac{a}{0.6}, \frac{b}{0.2}\right),\left(\frac{0}{0.08}, \frac{a}{0.08}, \frac{b}{0.7}\right)\right\rangle .
$$

Then $\alpha=\left\langle H, \mu_{\alpha}, \gamma_{\alpha}\right\rangle$ is an intuitionistic fuzzy hyper Ksubalgebra of $H$ (see [6]). It can be easily check that $\alpha=\left\langle H, \mu_{\alpha}, \gamma_{\alpha}\right\rangle$ is also an intuitionistic fuzzy strong hyper K-subalgebra of $H$.

Proposition 3.5. Every intuitionistic fuzzy strong hyper Ksubalgebra $\alpha=\left\langle H, \mu_{\alpha}, \gamma_{\alpha}\right\rangle$ satisfies the following assertions:

$$
(\forall x \in H)\left(\mu_{\alpha}(0) \geq \mu_{\alpha}(x) \& \gamma_{\alpha}(0) \leq \gamma_{\alpha}(x)\right) .
$$

Proof. Since $x \prec x$ for all $x \in H$, we have $0 \in x \circ x$. Hence $\mu_{\alpha}(0) \geq \inf _{z \in x \circ x} \mu_{\alpha}(z) \geq \mu_{\alpha}(x)$ and $\gamma_{\alpha}(0) \leq$ $\sup _{z \in x \circ x} \gamma_{\alpha}(z) \leq \gamma_{\alpha}(x)$. This completes the proof.

Theorem 3.6. Every intuitionistic fuzzy strong hyper Ksubalgebra is an intuitionistic fuzzy hyper K-subalgebra.

Proof. Let $\alpha=\left\langle H, \mu_{\alpha}, \gamma_{\alpha}\right\rangle$ be an intuitionistic fuzzy strong hyper K-subalgebra of $H$ and let $x, y \in H$. Then

$$
\begin{aligned}
& \inf _{z \in x \circ y} \mu_{\alpha}(z) \geq \mu_{\alpha}(x) \geq \min \left\{\mu_{\alpha}(x), \mu_{\alpha}(y)\right\}, \\
& \sup _{z \in x \circ y} \gamma_{\alpha}(z) \leq \gamma_{\alpha}(x) \leq \max \left\{\gamma_{\alpha}(x), \gamma_{\alpha}(y)\right\}
\end{aligned}
$$

for all $x, y \in H$. Hence $\alpha=\left\langle H, \mu_{\alpha}, \gamma_{\alpha}\right\rangle$ is an intuitionistic fuzzy hyper $\mathrm{K}$-subalgebra of $H$. 
The converse of Theorem 3.6 is not true as seen in the following example.

Example 3.7. Let $H=\{0, a, b\}$ be a hyper K-algebra with the following Cayley table:

\begin{tabular}{c|ccc}
$\circ$ & 0 & $a$ & $b$ \\
\hline 0 & $\{0\}$ & $\{0, a, b\}$ & $\{0, a, b\}$ \\
$a$ & $\{a\}$ & $\{0, a, b\}$ & $\{0, a, b\}$ \\
$b$ & $\{b\}$ & $\{a, b\}$ & $\{0, a, b\}$
\end{tabular}

Define an IFS $\alpha=\left\langle H, \mu_{\alpha}, \gamma_{\alpha}\right\rangle$ in $H$ by

$$
\alpha=\left\langle H,\left(\frac{0}{0.9}, \frac{a}{0.3}, \frac{b}{0.3}\right),\left(\frac{0}{0.09}, \frac{a}{0.7}, \frac{b}{0.7}\right)\right\rangle .
$$

Then $\alpha=\left\langle H, \mu_{\alpha}, \gamma_{\alpha}\right\rangle$ is an intuitionistic fuzzy hyper $\mathbf{K}$ subalgebra of $H$. But it is not an intuitionistic fuzzy strong hyper K-subalgebra of $H$ since $\inf _{z \in 0 \circ a} \mu_{\alpha}(z)=0.3<$ $0.9=\mu_{\alpha}(0)$ and $/$ or $\sup _{z \in 0 \circ a} \gamma_{\alpha}(z)=0.7>0.09=\gamma_{\alpha}(0)$.

Combining Lemma 3.2 and Theorem 3.6, we have

Theorem 3.8. If an IFS $\alpha=\left\langle H, \mu_{\alpha}, \gamma_{\alpha}\right\rangle$ in $H$ is an intuitionistic fuzzy strong hyper $\mathrm{K}$-subalgebra of $H$, then the nonempty intuitionistic level set $H_{\alpha}^{(m, n)}$ is a hyper $\mathrm{K}$ subalgebra of $H$ for all $m, n \in[0,1]$ with $m+n \leq 1$.

Theorem 3.9. Let $S$ be a subset of $H$ and let $\alpha(S)=$ $\left\langle H, \mu_{\alpha(S)}, \gamma_{\alpha(S)}\right\rangle$ be an IFS in $H$ defined by

$$
\begin{aligned}
& \mu_{\alpha(S)}(x):= \begin{cases}t_{1} & \text { if } x \in S, \\
t_{2} & \text { otherwise, }\end{cases} \\
& \gamma_{\alpha(S)}(x):= \begin{cases}s_{1} & \text { if } x \in S, \\
s_{2} & \text { otherwise, }\end{cases}
\end{aligned}
$$

for all $x \in H$, where $t_{1}, t_{2}, s_{1}, s_{2} \in[0,1]$ with $t_{1}>t_{2}$, $s_{1}<s_{2}, t_{i}+s_{i} \leq 1$ for $i=1,2$. Then

(i) $\alpha(S)=\left\langle H, \mu_{\alpha(S)}, \gamma_{\alpha(S)}\right\rangle$ is an intuitionistic fuzzy hyper $\mathrm{K}$-subalgebra of $H$ if and only if $S$ is a hyper K-subalgebra of $H$.

(ii) $H_{\alpha(S)}:=\left\{x \in H \quad H \quad \mu_{\alpha(S)}(x)=\right.$ $\left.\mu_{\alpha(S)}(0) \& \gamma_{\alpha(S)}(x)=\gamma_{\alpha(S)}(0)\right\}=S$.

Proof. (i) Assume that $\alpha(S)=\left\langle H, \mu_{\alpha(S)}, \gamma_{\alpha(S)}\right\rangle$ is an intuitionistic fuzzy hyper K-subalgebra of $H$ and let $x, y \in$ $S$. Then $\mu_{\alpha(S)}(x)=t_{1}=\mu_{\alpha(S)}(y)$ and $\gamma_{\alpha(S)}(x)=s_{1}=$ $\gamma_{\alpha(S)}(y)$. For any $a \in x \circ y$, we have

$\mu_{\alpha(S)}(a) \geq \inf _{u \in x \circ y} \mu_{\alpha(S)}(u) \geq \min \left\{\mu_{\alpha(S)}(x), \mu_{\alpha(S)}(y)\right\}=t_{1}$,

$\gamma_{\alpha(S)}(a) \leq \sup _{v \in x \circ y} \gamma_{\alpha(S)}(v) \leq \max \left\{\gamma_{\alpha(S)}(x), \gamma_{\alpha(S)}(y)\right\}=s_{1}$

Thus $\mu_{\alpha(S)}(a)=t_{1}$ and $\gamma_{\alpha(S)}(a)=s_{1}$, and so $a \in S$. Therefore $x \circ y \subseteq S$, and $S$ is a hyper K-subalgebra of $H$ by Lemma 2.1. Conversely, suppose that $S$ is a hyper Ksubalgebra of $H$ and let $x, y \in H$. If $x \notin S$ or $y \notin S$, then clearly

$$
\begin{aligned}
& \inf _{a \in x \circ y} \mu_{\alpha(S)}(a) \geq t_{2}=\min \left\{\mu_{\alpha(S)}(x), \mu_{\alpha(S)}(y)\right\}, \\
& \sup _{b \in x \circ y} \gamma_{\alpha(S)}(b) \leq s_{2}=\max \left\{\gamma_{\alpha(S)}(x), \gamma_{\alpha(S)}(y)\right\} .
\end{aligned}
$$

Assume that $x \in S$ and $y \in S$. Then $x \circ y \subseteq S$. Thus

$$
\begin{aligned}
& \inf _{a \in x \circ y} \mu_{\alpha(S)}(a)=t_{1}=\min \left\{\mu_{\alpha(S)}(x), \mu_{\alpha(S)}(y)\right\}, \\
& \sup _{b \in x \circ y} \gamma_{\alpha(S)}(b)=s_{1}=\max \left\{\gamma_{\alpha(S)}(x), \gamma_{\alpha(S)}(y)\right\} .
\end{aligned}
$$

Therefore $\alpha(S)=\left\langle H, \mu_{\alpha(S)}, \gamma_{\alpha(S)}\right\rangle$ is an intuitionistic fuzzy hyper K-subalgebra of $H$.

(ii) Straightforward.

Corollary 3.10. Let $\alpha(S)=\left\langle H, \mu_{\alpha(S)}, \gamma_{\alpha(S)}\right\rangle$ be defined as in Theorem 3.9. If it is an intuitionistic fuzzy strong hyper K-subalgebra of $H$, then $S$ is a hyper K-subalgebra of $H$.

Proof. It follows directly from Theorems 3.6 and 3.9 .

Definition 3.11. [5] Let $H_{1}$ and $H_{2}$ be hyper K-algebras. A mapping $f: H_{1} \rightarrow H_{2}$ is called a weak homomorphism if it satisfies:

(i) $f(0)=0$,

(ii) $\left(\forall x, y \in H_{1}\right)(f(x \circ y) \subseteq f(x) \circ f(y))$.

Theorem 3.12. Let $f: H_{1} \rightarrow H_{2}$ be a weak homomorphism of hyper $\mathrm{K}$-algebras. If $\beta=\left\langle H_{2}, \mu_{\beta}, \gamma_{\beta}\right\rangle$ is an intuitionistic fuzzy strong hyper $\mathrm{K}$-subalgebra of $\mathrm{H}_{2}$, then $\beta_{f}=\left\langle H_{1}, \mu_{\beta_{f}}, \gamma_{\beta_{f}}\right\rangle$ is an intuitionistic fuzzy hyper K-subalgebra of $H_{1}$, where $\mu_{\beta_{f}}$ and $\gamma_{\beta_{f}}$ are defined by $\mu_{\beta_{f}}(x)=\mu_{\beta}(f(x))$ and $\gamma_{\beta_{f}}(x)=\gamma_{\beta}(f(x))$ for all $x \in H_{1}$.

Proof. For any $x, y \in H_{1}$, we have

$$
\begin{aligned}
\inf _{z \in x \circ y} \mu_{\beta_{f}}(z) & =\inf _{z \in x \circ y} \mu_{\beta}(f(z)) \geq \inf _{f(z) \in f(x \circ y)} \mu_{\beta}(f(z)) \\
& \geq \inf _{f(z) \in f(x) \circ f(y)} \mu_{\beta}(f(z)) \geq \mu_{\beta}(f(x)) \\
& \geq \min \left\{\mu_{\beta}(f(x)), \mu_{\beta}(f(y))\right\} \\
& \geq \min \left\{\mu_{\beta_{f}}(x), \mu_{\beta_{f}}(y)\right\}, \\
\sup _{z \in x \circ y} \gamma_{\beta_{f}}(z) & =\sup _{z \in x \circ y} \gamma_{\beta}(f(z)) \leq \sup _{f(z) \in f(x \circ y)} \gamma_{\beta}(f(z)) \\
& \leq \sup _{f(z) \in f(x) \circ f(y)} \gamma_{\beta}(f(z)) \leq \gamma_{\beta}(f(x)) \\
& \leq \max \left\{\gamma_{\beta}(f(x)), \gamma_{\beta}(f(y))\right\} \\
& \leq \max \left\{\gamma_{\beta_{f}}(x), \gamma_{\beta_{f}}(y)\right\} .
\end{aligned}
$$

Hence $\beta_{f}=\left\langle H_{1}, \mu_{\beta_{f}}, \gamma_{\beta_{f}}\right\rangle$ is an intuitionistic fuzzy hyper $\mathrm{K}$-subalgebra of $H_{1}$. 
Let $\left(H_{1}, o_{1}, 0_{1}\right)$ and $\left(H_{2}, o_{2}, 0_{2}\right)$ be hyper K-algebras. Define a hyper operation $\circ$ on the product $\mathrm{H}_{1} \times \mathrm{H}_{2}$ as follows:

$$
\left(a_{1}, a_{2}\right) \circ\left(b_{1}, b_{2}\right)=\left(a_{1} \circ_{1} b_{1}, a_{2} \circ_{2} b_{2}\right)
$$

for all $\left(a_{1}, a_{2}\right),\left(b_{1}, b_{2}\right) \in H_{1} \times H_{2}$, where for $A \subseteq H_{1}$ and $B \subseteq H_{2}$ by $(A, B)$ we mean

$$
(A, B)=\{(a, b) \mid a \in A, b \in B\}, 0=\left(0_{1}, 0_{2}\right)
$$

and $\left(a_{1}, a_{2}\right) \prec\left(b_{1}, b_{2}\right) \Leftrightarrow a_{1} \prec b_{1}, a_{2} \prec b_{2}$. Then $\left(H_{1} \times H_{2}, 0,0\right)$ is a hyper $\mathrm{K}$-algebra (see [3]).

Theorem 3.13. If $\alpha_{1}=\left\langle H_{1}, \mu_{\alpha_{1}}, \gamma_{\alpha_{1}}\right\rangle$ and $\alpha_{2}=$ $\left\langle H_{2}, \mu_{\alpha_{2}}, \gamma_{\alpha_{2}}\right\rangle$ are intuitionistic fuzzy hyper $\mathrm{K}$ subalgebras of hyper K-algebras $\left(H_{1}, O_{1}, O_{1}\right)$ and $\left(H_{2}, \mathrm{o}_{2}, 0_{2}\right)$, respectively, then $\alpha_{1} \times \alpha_{2}=\left\langle H_{1} \times\right.$ $\left.H_{2}, \mu_{\alpha_{1} \times \alpha_{2}}, \gamma_{\alpha_{1} \times \alpha_{2}}\right\rangle$ is an intuitionistic fuzzy hyper $\mathrm{K}$ subalgebra of $\left(H_{1} \times H_{2}, 0,0\right)$.

Proof. For any $\left(x_{1}, x_{2}\right),\left(y_{1}, y_{2}\right) \in H_{1} \times H_{2}$, we have

$$
\begin{aligned}
& \inf _{\left(a_{1}, a_{2}\right) \in\left(x_{1}, x_{2}\right) \circ\left(y_{1}, y_{2}\right)} \mu_{\alpha_{1} \times \alpha_{2}}\left(a_{1}, a_{2}\right) \\
& =\inf _{\substack{\alpha_{1} \in x_{1} \circ_{1} y_{1} \\
\alpha_{2} \in x_{2} \alpha_{2} y_{2}}} \min \left\{\mu_{\alpha_{1}}\left(a_{1}\right), \mu_{\alpha_{2}}\left(a_{2}\right)\right\} \\
& =\min \left\{\inf _{\substack{a_{1} \in x_{1} o_{1} y_{1} \\
\mu_{\alpha_{1}}}} \mu_{\alpha_{1}}\left(a_{1}\right), \inf _{a_{2} \in x_{2} \circ_{2} y_{2}} \mu_{\alpha_{2}}\left(a_{2}\right)\right\} \\
& \geq \min \left\{\min \left\{\mu_{\alpha_{1}}\left(x_{1}\right), \mu_{\alpha_{1}}\left(y_{1}\right)\right\},\right. \\
& \left.\min \left\{\mu_{\alpha_{2}}\left(x_{2}\right), \mu_{\alpha_{2}}\left(y_{2}\right)\right\}\right\} \\
& =\min \left\{\min \left\{\mu_{\alpha_{1}}\left(x_{1}\right), \mu_{\alpha_{2}}\left(x_{2}\right)\right\},\right. \\
& \left.\min \left\{\mu_{\alpha_{1}}\left(y_{1}\right), \mu_{\alpha_{2}}\left(y_{2}\right)\right\}\right\} \\
& =\min \left\{\mu_{\alpha_{1} \times \alpha_{2}}\left(x_{1}, x_{2}\right), \mu_{\alpha_{1} \times \alpha_{2}}\left(y_{1}, y_{2}\right)\right\},
\end{aligned}
$$

$$
\begin{aligned}
& \quad \sup _{\left(a_{1}, a_{2}\right) \in\left(x_{1}, x_{2}\right) \circ\left(y_{1}, y_{2}\right)} \gamma_{\alpha_{1} \times \alpha_{2}}\left(a_{1}, a_{2}\right) \\
& =\sup _{\substack{a_{1} \in x_{1} 0_{1} y_{1} \\
a_{2} \in x_{2} \circ_{2} y_{2}}} \max \left\{\gamma_{\alpha_{1}}\left(a_{1}\right), \gamma_{\alpha_{2}}\left(a_{2}\right)\right\} \\
& =\max \left\{\sup _{a_{1} \in x_{1} \circ_{1} y_{1}} \gamma_{\alpha_{1}}\left(a_{1}\right), \sup _{a_{2} \in x_{2} \circ_{2} y_{2}} \gamma_{\alpha_{2}}\left(a_{2}\right)\right\} \\
& \leq \max \left\{\max \left\{\gamma_{\alpha_{1}}\left(x_{1}\right), \gamma_{\alpha_{1}}\left(y_{1}\right)\right\}\right. \\
& \left.\quad \max \left\{\gamma_{\alpha_{2}}\left(x_{2}\right), \gamma_{\alpha_{2}}\left(y_{2}\right)\right\}\right\} \\
& =\max \left\{\max \left\{\gamma_{\alpha_{1}}\left(x_{1}\right), \gamma_{\alpha_{2}}\left(x_{2}\right)\right\},\right. \\
& \left.\max \left\{\gamma_{\alpha_{1}}\left(y_{1}\right), \gamma_{\alpha_{2}}\left(y_{2}\right)\right\}\right\} \\
& =\max \left\{\gamma_{\alpha_{1} \times \alpha_{2}}\left(x_{1}, x_{2}\right), \gamma_{\alpha_{1} \times \alpha_{2}}\left(y_{1}, y_{2}\right)\right\} .
\end{aligned}
$$

Hence $\alpha_{1} \times \alpha_{2}=\left\langle H_{1} \times H_{2}, \mu_{\alpha_{1} \times \alpha_{2}}, \gamma_{\alpha_{1} \times \alpha_{2}}\right\rangle$ is an intuitionistic fuzzy hyper K-subalgebra of $\left(H_{1} \times H_{2}, \circ, 0\right)$.

Corollary 3.14. If $\alpha_{1}=\left\langle H_{1}, \mu_{\alpha_{1}}, \gamma_{\alpha_{1}}\right\rangle$ and $\alpha_{2}=$ $\left\langle H_{2}, \mu_{\alpha_{2}}, \gamma_{\alpha_{2}}\right\rangle$ are intuitionistic fuzzy strong hyper K-subalgebras of hyper $\mathbf{K}$-algebras $\left(H_{1}, O_{1}, O_{1}\right)$ and $\left(H_{2}, o_{2}, 0_{2}\right)$, respectively, then $\alpha_{1} \times \alpha_{2}=\left\langle H_{1} \times\right.$ $\left.H_{2}, \mu_{\alpha_{1} \times \alpha_{2}}, \gamma_{\alpha_{1} \times \alpha_{2}}\right)$ is an intuitionistic fuzzy (strong) hyper K-subalgebra of $\left(H_{1} \times H_{2}, \circ, 0\right)$.

Proof. Straightforward.

\section{References}

[1] A. Borumand Saeid, R. A. Borzoei and M. M. Zahedi, (Weak) implicative hyper $K$-ideals, Bull. Korean Math. Soc. 40 (1995), no. 1, 123-137.

[2] R. A. Borzoei and Y. B. Jun, Intuitionistic fuzzifications of (weak) implicative hyper K-ideals in hyper K-algerbas, Iranian J. Fuzzy Systems (submitted).

[3] R. A. Borzoei, A. Hasankhani, M. M. Zahedi and Y. B. Jun, On hyper $K$-algebras, Math. Japon. 52 (2000), no. 1, 113-121.

[4] R. A. Borzoei and Y. B. Jun, Intuitionistic fuzzy hyper $B C K$-ideals of hyper BCK-algerbas, Iran. J. Fuzzy Systems 1 (2004), no. 1, 65-78.

[5] Y. B. Jun, On fuzzy hyper $K$-subalgebras of hyper $K$-algebras, Sci. Math. 3 (2000), no. 1, 67-76.

[6] Y. B. Jun and C. H. Park, Intuitionistic fuzziness of strong hyper $K$-ideals and hyper $K$-subalgebras in hyper K-algebras, J. Appl. Math. Inform. (submitted).

[7] Y. B. Jun and E. H. Roh, On strong hyper K-ideals of hyper $K$-algebras, Ital. J. Pure Appl. Math. No. 10 (2001), 79-84.

[8] Y. B. Jun and E. H. Roh, Fuzzy (weak) implicative $K$-ideals, Bull. Korean Math. Soc. 43 (2006), no. 1, 141-148.

[9] Y. B. Jun and W. H. Shim, Fuzzy positive implicative hyper $K$-ideals of hyper $K$-algebras, Honam Math. J. 25 (2003), no. 1, 43-52.

[10] Y. B. Jun and W. H. Shim, Fuzzy hyper $K$-ideals of hyper $K$-algebras, J. Fuzzy Math. 12 (2004), no. 4, 861-871.

[11] Y. B. Jun, M. M. Zahedi, X. L. Xin and R. A. Borzoei, On hyper BCK-algebras, Ital. J. Pure Appl. Math. No. 8 (2000), 127-136.

[12] F. Marty, Sur une generalization de la notion de groupe, 8th Congress Math. Scandinaves, Stockholm (1934), 45-49.

[13] M. M. Zahedi, R. A. Borzoei, Y. B. Jun and A. Hasankhani, Some results on hyper $K$-algebras, Sci. Math. 3 (2000), no. 1, 53-59.

\section{저 자 소 개}

Chul Hwan Park

제17권 1호 참조

E-mail : chpark@ulsan.ac.kr 